\section{Neurobiological basis of psychopathy}

\author{
R. JAMES R. BLAIR
}

To understand a psychiatric disorder we need to know why the pathology causes the behavioural disturbance, the neural structures implicated in the pathology and the cause of the dysfunction in the neural structures. With regard to psychopathy, we have clear indications regarding why the pathology gives rise to the emotional and behavioural disturbance and important insights into the neural systems implicated in this pathology. What remains unclear is why these neural systems are dysfunctional.

\section{WHAT IS PSYCHOPATHY?}

Psychopathy is a disorder, defined by Hare's Psychopathy Checklist - Revised (PCL-R) and characterised in part by a diminished capacity for remorse and poor behavioural controls (Hare, 1991). Classifications of psychopathy are not synonymous with diagnoses of conduct disorder or antisocial personality disorder (American Psychiatric Association, 1994) but represent an extension. These psychiatric diagnoses are poorly specified and concentrate on the antisocial behaviour. Because of this imprecision, the diagnostic rate of conduct disorder is $16 \%$ of boys in mainstream education (American Psychiatric Association, 1994) and over $80 \%$ for antisocial personality disorder in adult forensic institutions (Hart \& Hare, 1996). In contrast, psychopathy is defined not only by antisocial behaviour but also by emotional impairment such as the lack of guilt. Only onethird of those who are diagnosed with antisocial personality disorder meet criteria for psychopathy (Hart \& Hare, 1996). Moreover, a diagnosis of psychopathy, unlike antisocial personality disorder, is informative regarding a patient's future risk (Hare, 1991).

A striking feature of much of the antisocial behaviour shown by individuals with psychopathy is that it is mostly instrumental in nature, i.e. goal-directed towards achieving money, sexual opportunities or increased status (Cornell et al, 1996). This suggests that the pathology associated with psychopathy interferes with socialisation. It is well known that empathy-inducing, positive parenting practices give rise to less antisocial behaviour than punishmentbased, negative parenting practices. This relationship is shown in healthy developing children as well as in children with conduct disorder who do not present with the emotional dysfunction of psychopathy. However, for children with conduct disorder who do present with emotional dysfunction, there is no relationship between parenting behaviours and level of antisocial behaviour (Wootton et al, 1997). In other words, the emotional impairment found in individuals with psychopathy interferes with socialisation such that the individual does not learn to avoid antisocial behaviour.

Socialisation involves aversive conditioning and instrumental learning. In order to learn that hitting another is bad, this thought must be associated with an aversive unconditioned stimulus (e.g. the distress of the victim). Similarly, learning to avoid committing moral transgressions involves committing a moral transgression and then being 'punished' by the aversive response of the victim's distress (Blair, 1995). Individuals with psychopathy present with severe difficulties in both aversive conditioning and instrumental learning (Patrick, 1994; Blair, 2001). Moreover, they have particular difficulties processing the fearfulness and sadness of others (Blair, 2001).

\section{THE NEURAL BASIS OF PSYCHOPATHY}

The amygdala is involved in aversive conditioning and instrumental learning (LeDoux, 1998). It is also involved in the response to fearful and sad facial expressions (Blair et al, 1999). The amygdala is thus involved in all the processes that, when impaired, give rise to the functional impairments shown by individuals with psychopathy. It is therefore suggested that amygdala dysfunction is one of the core neural systems implicated in the pathology of psychopathy (Patrick, 1994; Blair et al, 1999).

Interestingly, two recent neuroimaging studies have confirmed that amygdala dysfunction is associated with psychopathy (Tiihonen et al, 2000; Kiehl et al, 2001). Thus, Tiihonen et al (2000) used volumetric magnetic resonance imaging (MRI) to explore the relationship between amygdaloid volume and degree of psychopathy in violent offenders as measured by the PCL-R. They found that high levels of psychopathy were associated with reduced amygdaloid volume. Kiehl et al (2001) used functional MRI to examine neural responses in individuals with high $(>28 / 40)$ and low $(<23 / 40)$ scores on the PCL-R during an emotional memory task where the participant processed words of neutral and negative valence. Kiehl et al found a reduced amygdala response in the highscoring group, relative to the low-scoring group, during the processing of words of negative valence.

There have been suggestions that other neural systems are dysfunctional in individuals with psychopathy. Thus, on the basis of neuropsychological and neuroimaging findings for violent offenders, it has been argued that the frontal cortex could be dysfunctional (Morgan \& Lilienfield, 2000; Soderstrom et al, 2000). However, all of these studies, with the exception of one by (Raine et al, 2000), have been with violent offenders rather than individuals with psychopathy. This is noteworthy as there are crucial differences between the general population of violent offenders and these individuals. Indeed, neuropsychological work with individuals with psychopathy, unlike work with individuals who are violent, has repeatedly found frontal functioning to be intact (Kandel \& Freed, 1989).

The volumetric MRI study conducted by Raine et al (2000) did assess individuals scoring highly on the PCL-R. This study reported reduced prefrontal grey, but not white, matter volume in these individuals. However, Raine et al were unable to differentiate between grey matter from different regions of the frontal cortex. This is unfortunate, as although it appears clear 
that there is no generalised frontal cortical dysfunction in individuals with psychopathy there is one region of the frontal cortex that could be impaired, i.e. the orbitofrontal cortex (OFC).

The OFC, especially the medial OFC, receives extensive projections from, and sends extensive projections to, the amygdala. Moreover, the medial OFC is involved in instrumental learning and response reversal - both of which functions are impaired in individuals with psychopathy (LaPierre et al, 1995). In addition, there have been attempts to link the disorder of psychopathy with the neurological condition of 'acquired sociopathy' following lesions of the OFC (Anderson et al, 1999). Patients with 'acquired sociopathy' can present with socially inappropriate behaviour and aggression following their lesion. However, if aggression is seen it is almost exclusively reactive (i.e. frustration/threat-induced) in nature and not the instrumental goal-directed aggression shown by individuals with psychopathy. Indeed, it has been argued that the extensive damage seen in those patients with 'acquired sociopathy' who present with reactive aggression includes regions of the lateral OFC that are implicated in modulating basic brain-stem mechanisms that mediate the fight-flight response to threat (Blair, 2001). This modulatory function, and by implication this region, of the OFC is not thought to be dysfunctional in individuals with psychopathy.

Thus, it is suggested that the neural structures implicated in psychopathic pathology include the amygdala and OFC. The basic causes of the pathology remain unclear; however, there are interesting possibilities. One is that pathology in the noradrenergic system can lead to the observed amygdala dysfunction. Certainly, administration of the $\beta$-adrenergic blocker propranolol, as well as amygdala damage, blocks the improvement in episodic memory for emotionally arousing events, as well as disrupting the processing of sad facial expressions (Harmer et al, 2001). A second possibility concerns the potential value of genetic information and mouse models. Many mouse models of aggression concentrate on a heightened reactively aggressive response to an intruder. However, a recent mouse model has been developed without a functional 'tailless' protein. The gene tailless is a member of the superfamily of genes that encode transcription factors of the ligand-activated nuclear

R. JAMES R. BLAIR, PhD, Unit on Affective Cognitive Neuroscience, Mood and Anxiety Program, National Institute of Mental Health, I5K North Drive, Bethesda, MD 20892, USA

(First received 10 December 200I, final revision 15 May 2002, accepted 16 May 2002)

receptor type, and is expressed in the invertebrate and vertebrate brain. Interestingly, mice lacking a functional tailless protein show reduced amygdaloid volume. Moreover, they show heightened aggression even to litter-mates and the females lack normal maternal instincts (Monaghan et al, 1997). Although it is difficult currently to extrapolate from these data to an understanding of psychopathy, the study of such animals should allow a molecular approach to understanding the genetic architecture of the forebrain. These data will undoubtedly be invaluable in furthering our understanding of psychopathy.

It is important to make one final point. The lifestyle of the individual with psychopathy may exacerbate any neurobiological impairments. One feature associated with psychopathy is substance misuse. This could be contributing to apparent impairments. For example chronic amphetamine misuse has been shown to lead to disturbance in functions mediated by the OFC (Rogers et al, 1999). In this context, it is interesting to note that although performance on measures thought to require amygdala involvement is impaired substantially in both adults with psychopathy and children with psychopathic tendencies, performance on measures thought to require the OFC is impaired substantially only in the adults (Blair et al, 2001). This, of course, could reflect the developmental course of the disorder. However, alternatively, it could reflect the lifestyle of the individual with psychopathy where substance misuse has interacted with the fundamental pathology to produce additional OFC pathology.

\section{CONCLUSIONS}

The suggestion is that the pathology of individuals with psychopathy detrimentally affects two processes that are required for socialisation, i.e. aversive conditioning and instrumental learning. This is thought to result from amygdala dysfunction potentially compounded by OFC dysfunction. However, the reasons for this dysfunction remain unknown. The noradrenergic system could be implicated but why this system should be dysfunctional is, again, unknown. An answer is likely to follow an understanding of the genetic architecture and morphogenesis of the forebrain.

\section{REFERENCES}

\section{American Psychiatric Association (1994)}

Diagnostic and Statistical Manual of Mental Disorders (4th edn) (DSM-IV). Washington, DC: APA.

Anderson, S. W., Bechara, A., Damasio, H., et al (1999) Impairment of social and moral behaviour related to early damage in human prefrontal cortex. Nature Neuroscience, 2, 1032-1037.

Blair, R. J. R. (1995) A cognitive developmental approach to mortality: investigating the psychopathy. Cognition, 57, I-29.

- (200I) Neuro-cognitive models of aggression, the Antisocial Personality Disorders and Psychopathy. Journal of Neurology, Neurosurgery and Psychiatry, 7I, 727-731.

_ , Morris, J. S., Frith, C. D., et al (1999) Dissociable neural responses to facial expressions of sadness and anger. Brain, 122, 883-893.

_ , Colledge, E. \& Mitchell, D. (200I) Somatic markers and response reversal: is there orbitofrontal cortex dysfunction in boys with psychopathic tendencies? Journal of Abnormal Child Psychology, 29, $499-511$.

Cornell, D. G., Warren, J., Hawk, G., et al (1996) Psychopathy in instrumental and reactive violent offenders. Journal of Consulting and Clinical Psychology, 64, 783-790.

Hare, R. D. (199I) The Hare Psychopathy Checklist Revised. Toronto, Ontario: Multi-Health Systems.

Harmer, C. J., Perrett, D. I., Cowen, P. J., et al (200I) Administration of the beta-adrenoceptor blocker propranolol impairs the processing of facial expressions of sadness. Psychopharmacology (Berlin), 154, 383-389.

Hart, S. D. \& Hare, R. D. (1996) Psychopathy and antisocial personality disorder. Current Opinion in Psychiatry, 9, 129-132.

Kandel, E. \& Freed, D. (1989) Frontal lobe dysfunction and antisocial behavior: a review. Journal of Clinical Psychology, 45, 404-413.

Kiehl, K. A., Smith, A. M., Hare, R. D., et al (200I) Limbic abnormalities in affective processing by criminal psychopaths as revealed by functional magnetic resonance imaging. Biological Psychiatry, 50, 677-684.

LaPierre, D., Braun, C. M. J. \& Hodgins, S. (1995) Ventral frontal deficits in psychopathy: Neuropsychological test findings. Neuropsychologia, 33 |39-151.

LeDoux, J. (1998) The Emotional Brain. New York: Weidenfeld \& Nicolson. 
Monaghan, A. P., Bock, D., Gass, P., et al (1997)

Defective limbic system in mice lacking the tailless gene. Nature, 390, 515-517.

Morgan, A. B. \& Lilienfield, S. O. (2000) A metaanalytic review of the relation between antisocial behavior and neuropsychological measures of executive function. Clinical Psychology Review, 20, I13-136.

Patrick, C. J. (1994) Emotion and psychopathy: startling new insights. Psychophysiology, 31, 319-330.

Raine, A., Lencz, T., Bihrle, S., et al (2000) Reduced prefrontal gray matter volume and reduced autonomic activity in antisocial personality disorder. Archives of General Psychiatry, 57, 119-127.

Rogers, R. D., Everitt, B. J., Baldacchino, A., et al (1999) Dissociable deficits in the decision-making cognition of chronic amphetamine abusers, opiate abusers, patients with focal damage to prefrontal cortex, and tryptophan-depleted normal volunteers: evidence for monoaminergic mechanisms. Neuropsychopharmacology, 20, 322-339.

Soderstrom, H., Tullberg, M., Wikkelso, C., et a (2000) Reduced regional cerebral blood flow in non-psychotic violent offenders. Psychiatry Research, 98, 29-4l.

Tiihonen, J., Hodgins, S., Vaurio, O., et al (2000) Amygdaloid volume loss in psychopathy. Society for Neuroscience Abstracts, 2017

Wootton, J. M., Frick, P. J., Shelton, K. K., et al (1997) Ineffective parenting and childhood conduct problems: the moderating role of callous-unemotional traits. Journal of Consulting and Clinical Psychology, 65, 292-300. 